\title{
ESTUDO GENÉTICO-CLÍNICO E CITOGENÉTICO DE CRIANÇAS AUTISTAS
}

\author{
CLINICAL, GENETIC AND CYTOGENETIC STUDY OF AUTISTIC CHILDREN
}

\author{
Greice A. Molfetta ${ }^{1}$; Têmis Maria Félix ${ }^{2}$ \& João M. Pina Neto 3
}

\begin{abstract}
Aluna do Curso de Pós-Graduação'; Docente ${ }^{3}$ do Departamento de Genética - Faculdade de Medicina de Ribeirão Preto da Universidade de São Paulo; Médica Assistente ${ }^{2}$ da Unidade de Genética Médica do Hospital de Clínicas de Porto Alegre.

CoRRESPONDÊNCIA: Greice A. Molfetta - Departamento de Genética - Faculdade de Medicina de Ribeirão Preto da USP - Av. Bandeirantes, 3900 - CEP: 14049-900 - Ribeirão Preto - SP. e-mail: gamolf@usp.br
\end{abstract}

MOLFETTA GA; FÉLIX TM \& PINA NETO JM. Estudo genético-clínico e citogenético de crianças autistas. Medicina, Ribeirão Preto, 30: 514-521, out./dez. 1997.

RESUMO: O autismo infantil é caracterizado pelo comportamento típico que pode ser causado por uma doença orgânica ou por um distúrbio emocional. Através de um estudo genético-clínico e citogenético, tivemos como objetivo detectar a presença de doenças orgânicas, principalmente de etiologia genética, que pudessem estar relacionadas com o quadro de autismo apresentado por dezessete meninos que freqüentavam a AMA de Ribeirão Preto. Concluímos que quatorze indivíduos não possuíam alterações orgânicas que pudessem estar relacionadas com o quadro clínico por eles apresentado; um indivíduo apresentou quadro clínico compatível com macrocefalia; um indivíduo apresentou quadro clínico compatível com uma nova síndrome de deficiência mental, ligada ao $\mathrm{X}$, associada à macrossomia, macrocefalia e obesidade, e um indivíduo apresentou a Síndrome de Angelman (SA). O estudo citogenético mostrou-se normal para todos os indivíduos estudados, assim como a pesquisa de fragilidade Xq27.3, excluindo a todos da possibilidade de apresentarem a Síndrome do X-frágil. O estudo molecular, específico para a detecção da SA, revelou a presença, no paciente, de herança biparental para os marcadores utilizados; como a clínica desse paciente é extremamente sugestiva, concluímos estar frente a um caso de SA com herança biparental.

UNITERMOS: Autismo Infantil. Retardo Mental. Obesidade. Síndrome do X-Frágil. Síndrome de Angelman.

\section{INTRODUÇÃO}

Autismo Infantil é o quadro clássico, descrito por Kanner ${ }^{(1,2)}$, por isto também chamado de Síndrome Autista ou Autismo Infantil de Kanner. É definido por alterações presentes desde idades muito precoces e que se caracteriza, sempre, pela presença de desvios nas relações interpessoais, linguagem/comunicação e comportamento; tem duas características cardinais: isolamento extremo e um desejo ansiosamente obsessivo de preservação da rotina, a mesmice ${ }^{(3)}$.
O Autismo Infantil é um estado mórbido, crônico, com início sempre na infância, em geral até o final do $3^{\circ}$ ano de vida, que afeta meninos em uma proporção de quatro a seis para cada menina, e com prevalência de, pelo menos, 4:10000 ${ }^{(4)}$.

A Síndrome de Autismo Infantil não é mais considerada como entidade nosológica única, ou enfermidade com causa uniforme, e, sim, como uma síndrome comportamental de muitas etiologias, cujo início, no entanto, deve ser precoce. O autismo é uma síndrome comportamentalmente definida, que pode apresentar 
graus bastante variáveis de comprometimento, na qual, se pode ou não, demonstrar a presença de alguma condição neurológica, genética, metabólica, infecciosa ou emocional ${ }^{(4,5)}$.

Alguns autores utilizam o termo autista apenas naqueles casos que se enquadram rigidamente no quadro descrito por Kanner ${ }^{1}$, aqueles, nos quais não se encontra qualquer evidência de estado patológico associado aos sinais de autismo, e que, são os chamados casos de "autismo isolado"(4).

A suspeita de que fatores genéticos estejam envolvidos entre os agentes causais dos casos de autismo isolado apóiam-se em algumas evidências: concentração de casos em determinadas famílias, alta concordância em gêmeos monozigóticos e associação com alguns quadros sindrômicos de etiologia genética ${ }^{(4,6)}$.

Já foi notado que o autismo tem agregação familial, sendo consistente a observação de que cerca de 2-3\% das famílias apresentam, pelo menos, dois irmãos afetados. Apesar de baixo, este número é 50 a 100 vezes maior do que o da prevalência estimada para o autismo infantil, na população geral, que é de 4:10000, e é preciso salientar, também, que os afetados, geralmente, não deixam filhos ${ }^{(6,7)}$.

Evidências melhores para a importância de fatores genéticos são provenientes de estudos com gêmeos, onde a concordância é maior entre monozigóticos do que entre dizigóticos. Folstein \& Rutter, em $1977^{(8)}$, baseando-se na avaliação de 21 pares de gêmeos do mesmo sexo, nos quais, pelo menos um apresentava autismo, apontaram $36 \%$ de concordância em gêmeos monozigóticos e $0 \%$ em gêmeos dizigóticos. Ritvo et al., em $1985^{(9)}$, obtiveram diferenças significativas entre taxas de concordância dos gêmeos monozigóticos e dizigóticos, sendo a taxa de concordância para os monozigóticos de $96 \%$, e Bayler et al. ${ }^{(10)}$, encontraram $60 \%$ de concordância para os monozigóticos contra $0 \%$ para os dizigóticos.

Alguns quadros sindrômicos de etiologia cromossômica (Síndrome do Duplo Y e Síndrome do Triplo X), de etiologia monogênica (Síndrome do X-frágil, Síndrome de Rett, Esclerose Tuberosa, Mucopolissacaridose tipo III), de etiologia genética heterogênea (Síndrome de Angelman) ou de etiologia ambiental (efeitos fetais da rubéola, do tolueno), podem ocorrer com alguns sintomas de autismo; nesses casos, temos os "traços de autismo". Os sinais de autismo, que são encontrados nos quadros sindrômicos, estão ligados a uma ampla variedade de desordens cognitivas, sendo que esses déficits cognitivos incluem, normalmente, distúrbios de leitura, do soletrar e fonoarticulatórios, assim como atraso na aquisição da linguagem falada e deficiência mental ${ }^{(4,7)}$. Estas associações são eventuais e podem ter ocorrido por acaso, porém a freqüência com que os dois quadros têm sido encontrados em um mesmo indivíduo mostra que deve haver algum fator em comum a ambos os estados, ou que a presença de um deles facilita o desenvolvimento do outro $^{(11)}$.

A Síndrome do X-frágil é a mais estudada anomalia genética, relacionada com o autismo. Esta síndrome está presente em cerca de $10 \%$ dos indivíduos que freqüentam instituições para autistas e é responsável por cerca de $50 \%$ dos casos de deficiência mental ligada ao cromossomo $\mathrm{X}^{(12)}$. Estudos multicêntricos têm demonstrado uma prevalência da Síndrome do X-frágil de $2,4 \%$ a $15 \%$ em homens com comportamento autista $\left({ }^{13 / 15)}\right.$. Blomquist et al. ${ }^{(13,14,15)}$ estimaram a freqüência da Síndrome X-frágil em 102 crianças diagnosticadas como autistas e encontraram o $\mathrm{Fra}(\mathrm{X})$ em 13/83 meninos (16\%) e em 0/19 meninas. Tranebjaerg \& Kure ${ }^{(16)}$ estudaram 32 pacientes com diagnóstico de autismo e encontraram a presença do $\mathrm{Fra}(\mathrm{X})$ em 2/20 meninos (10\%) e em 0/12 meninas. Apesar de serem poucos os relatos da Síndrome do $\mathrm{X}$-frágil em mulheres autistas, Cohen et al. ${ }^{(17)}$ encontraram 4/33 (12,1\%) meninas com comportamento autista, que foram positivas para o $\mathrm{Fra}(\mathrm{X})$, indicando que a proporção da Síndrome do X-frágil, em mulheres, pode ser tão alta quanto em homens.

Os pacientes com a Síndrome do X-frágil apresentam uma série de sinais característicos do comportamento autista: ausência de contato visual, recusa ao contato físico, auto-agressão, comportamento indiferente e arredio, fala anormal e linguagem ecolálica e perseverativa para palavras ou frases ${ }^{(18)}$.

A pesquisa do $\mathrm{X}$-frágil é considerada obrigatória em indivíduos com deficiência mental de causa desconhecida e em autistas. Admite-se que, em $80 \%$ dos homens afetados, há deficiência mental e/ou manifestações autísticas, e que $30 \%$ das mulheres heterozigotas apresentam deficiência mental ou quadros autísticos, em geral, menos graves que os presentes nos homens ${ }^{(18)}$.

No presente trabalho, a partir do exame genético-clínico e citogenético, buscamos encontrar evidências de doenças orgânicas que pudessem estar relacionadas com o quadro de autismo e deficiência mental apresentado pelas crianças que se encontravam na AMA de Ribeirão Preto. 


\section{CASUÍSTICA E MÉTODOS}

\section{Casuística:}

Foi realizado o exame genético-clínico e citogenético em dezessete meninos que freqüentavam a AMA de Ribeirão Preto, durante o ano de 1995. Esses dezessete pacientes foram previamente diagnosticados como autistas pela equipe profissional da AMA de Ribeirão Preto, seguindo-se os critérios diagnósticos do DSMIII-R.

A idade dos indivíduos estudados variou de 6 a $19 \operatorname{anos}(\bar{x}=10,5)$; a idade das mães variou de 25 a 50 anos $(\bar{x}=28,6)$ e a idade dos pais variou de 26 a 57 $\operatorname{anos}(\bar{x}=36,8)$; nenhuma das famílias estudadas apresentou consangüinidade entre os pais; o número de gestações variou de 2 a $9(\bar{x}=2,8)$ e, em quatorze casos, a história familial foi negativa. Em dois casos, houve relatos de sobrinhos paternos frequientando a APAE, devido a dificuldade de aprendizado, e, em um caso, houve relato de parentes paternos com quadro de esquizofrenia.

\section{Métodos}

\section{MÉTodo Clínico}

A avaliação clínica desses pacientes foi realizada por anamnese, seguida de exame físico geral e dismorfológico, além de dados antropométricos, de uso genético-clínico e de exames complementares como: eletroencefalograma, exame radiológico e tomográfico do crânio, exame de triagem de erros inatos do metabolismo e exame radiológico de idade óssea.

\section{Método Laboratorial}

A) Citogenético: foi estudado o cariótipo com bandamento GTG, em sangue periférico de todos os pacientes, visando à detecção de alterações cromossômicas, e foi realizado o estudo citogenético específico, para evidenciar o sítio frágil do cromossomo $\mathrm{X}$ (Xq27.3). O estudo citogenético foi realizado através da cultura temporária de linfócitos, seguindo a técnica de Moorhead et al. ${ }^{(19)}$, modificada. A coloração das metáfases cromossômicas foi realizada, utilizando a técnica de bandamento GTG, segundo Scheres ${ }^{(20)}$. A análise do material foi realizada, baseando-se nos critérios estabelecidos para a preparação e análise do X-frágil $^{21}$. Para evidenciar o sítio frágil do cromossomo X, na região Xq27.3, dois sistemas de indução que interferem no metabolismo do folato foram utilizados ${ }^{(18)}$.

B) Molecular: foi realizado o estudo molecular específico para a detecção da SA, devido à hipótese diagnóstica, realizada em um dos pacientes dessa amostra. Esse estudo consta da análise do padrão de metilação da região $15 q 11-13$, através da técnica de Southern blotting, utilizando a sonda KB17 - que detecta o estado de metilação da ilha $\mathrm{CpG}$ do gene SNRPN - e as enzimas de restrição, sensíveis à metilação XbaI e NotI. Essa análise serve para o diagnóstico da SA a nível molecular ${ }^{(22)}$. Realizamos, também, a análise da segregação dos polimorfismos de repetição de (CA)n, através da técnica de PCR, utilizando os primers 196 (D15S113) e IR43R (D15S11); essa análise visa estabelecer a causa genética da $\mathrm{SA}^{(22)}$.

\section{RESULTADOS}

A partir do exame genético-clínico dos dezessete indivíduos que se encontravam na AMA de Ribeirão Preto, observamos que quatorze indivíduos $(82,3 \%)$ não possuíam alterações orgânicas ou genéticas compatíveis com a falta de aprendizado e distúrbio de comportamento apresentados; um indivíduo $(5,9 \%)$ apresentou quadro clínico compatível com macrocefalia não benigna (McKusick $\mathrm{n}^{\circ}$ 248000), outro indivíduo (5,9\%) apresentou uma nova síndrome de deficiência mental, ligada ao cromossomo $\mathrm{X}$, associada à macrossomia, macrocefalia e obesidade, descrita por Baraitser et al., em $1987^{(23)}$, e encontramos um caso $(5,9 \%)$, apresentando quadro clínico compatível com a Síndrome de Angelman. Em relação ao estudo citogenético, não foi detectada nenhuma anormalidade cromossômica, nem foi detectado algum caso de fragilidade cromossômica Xq27.3. O cariótipo com bandamento GTG foi normal para todos os casos (46, XY).

O caso diagnosticado como portador de macrocefalia (McKusick no 248000) é uma criança do sexo masculino, com 7 anos, na primeira consulta; filho de um casal jovem e não consangüíneo; peso ao nascimento $=4260 \mathrm{~g}($ acima P90), estatura $=53,0 \mathrm{~cm}($ acima P90) e perímetro cefálico $=37,0 \mathrm{~cm}($ acima P90); o desenvolvimento neuropsicomotor foi normal (firmou pescoço aos 2 meses, sentou com apoio aos 5 meses, sentou sem apoio aos 9 meses, andou com apoio aos 1 ano e 2 meses e andou sem apoio aos 1 ano e 4 meses); houve regressão da fala, as primeiras palavras foram aos 9 meses mas, atualmente, a criança não fala; prefere ficar isolada, não brincando com outras crianças; esporadicamente apresenta crise de risos (gargalhadas) e movimentos em círculo; é muito agitada; aos 2 anos, foi internada por desidratação. Ao exame: estatura $=134,5 \mathrm{~cm}(\mathrm{P} 75-\mathrm{P} 90)$; peso $=26600 \mathrm{~g}(\mathrm{P} 75-\mathrm{P} 90)$; perímetro cefálico $=56,0 \mathrm{~cm}($ acima $\mathrm{P} 97) ;$ mão 
$\mathrm{D}=14,5 \mathrm{~cm}$ (P75); dedo médio $\mathrm{D}=6,2 \mathrm{~cm}(\mathrm{P} 75)$; distância intercantal interna $=2,8 \mathrm{~cm}(\mathrm{P} 25-\mathrm{P} 50)$; distância intercantal externa $=8,5 \mathrm{~cm}(\mathrm{P} 50-\mathrm{P} 75)$; orelha $=5,4 \mathrm{~cm}$ (25-P50); pênis =5,5cm (P50-P90); macrocefalia, frouxidão ligamentar generalizada; exame neurológico sem alterações. Exames complementares: $\mathrm{EEG}=$ normal; CT de crânio $=$ normal; $\mathrm{RX}$ de crânio $=$ macrocrania com módulo aumentado: (16,93 - N:14,5-16,8); RX de idade óssea $=$ compatível com a idade cronológica.

$\mathrm{O}$ caso que apresenta síndrome de deficiência mental, ligada ao X com macrossomia, macrocefalia e obesidade (Figura 1 A e 1B), é uma criança do sexo masculino, com 10 anos, na primeira consulta; peso ao nascimento $=4005 \mathrm{~g}(\mathrm{P} 75-\mathrm{P} 90)$ e estatura $=55,0 \mathrm{~cm}$ (P97); a criança nasceu hipotônica, melhorando em seguida; apresentou desenvolvimento neuropsicomotor normal, apresentou apenas atraso de linguagem, iniciando as primeiras palavras aos 2 anos de idade e, ainda hoje, fala palavras mas não forma frases; na infância, apresentou somente varicela; negam cirurgias ou internações. Ao exame: estatura $=155 \mathrm{~cm}$ (acima P97, $\overline{\mathrm{x}}$ para 13 anos e 3 meses); peso $=58000 \mathrm{~g}$ (acima P97, $\bar{x}$ para 15 anos e 6 meses); perímetro cefálico $=61,5 \mathrm{~cm}$ (muito acima P97); mão direita $=16,6 \mathrm{~cm}$ (P75-P97); dedo médio direito = 7,0cm (P50-P75); distância intercantal interna $=3,5 \mathrm{~cm}$ (acima P97); distância intercantal externa $=9,5 \mathrm{~cm}$ (acima P97); orelha $=7,5 \mathrm{~cm}$ (acima P97); pênis = 4,5cm (P10-P50); testículo direito $=10 \mathrm{ml}$ (muito acima P90); testículo esquerdo $=8 \mathrm{ml}$ (muito acima P90); macrocefalia, obesidade, evita contato com olhar, micropênis aparente; prega palmar única, na mão direita. Exames complementares: CT de crânio = macrocrania, sem alterações do parênquima cerebral; RNM de crânio: normal; RX de crânio = normal, porém módulo craniano aumentado (18,6 - N: 14,1-17,0); RX de idade óssea = compatível com a idade cronológica.

O indivíduo portador da Síndrome de Angelman (Figuras 1C e 1D) é uma criança do sexo masculino, com 10 anos, na primeira consulta; filho de um casal jovem e não consangüíneo; a partir do $5^{\circ}$ mês, começou a apresentar crises convulsivas, que se tornaram freqüentes; apresentou atraso no desenvolvimento neuropsicomotor e deficiência mental; anda claudicando, com marcha de base alargada e aspecto de marionete, pela posição fletida dos braços e pernas; não tem controle de esfíncteres diurno e noturno, e não fala. Ao exame: estatura $=136,0 \mathrm{~cm}(\mathrm{P} 50)$; peso $=25000 \mathrm{~g}$ (P50); perímetro cefálico $=46,0 \mathrm{~cm}$ (abaixo P2); Índice Cefálico $=90,9$; boca $=5,5 \mathrm{~cm}($ acima de $+2 \mathrm{SD})$; ore- lha $=6,3 \mathrm{~cm}(\mathrm{P} 50-\mathrm{P} 75)$; pênis = 4,5cm (P10-P50); as demais características clínicas são mostradas na Tabela I.

Quanto ao estudo molecular específico para a detecção da SA, tanto a análise do padrão de metilação da região 15q11-13 como a análise dos polimorfismos de repetição de (CA)n mostraram herança biparental, isto é, a presença de ambos os alelos, materno e paterno (o estudo molecular foi descrito em Pina Neto et al. $\left.{ }^{(22)}\right)$.

\section{DISCUSSÃO}

O autismo é uma síndrome bastante heterogênea, tanto clínica como etiologicamente. Assim, torna-se importante a realização de estudos com o objetivo de esclarecer as causas deste estado patológico e tentar diagnosticar quais os casos clássicos de autismo e quais os casos de autismo associados a outros quadros patológicos. A partir desta diferenciação, busca-se a definição etiológica exata para cada caso observado,

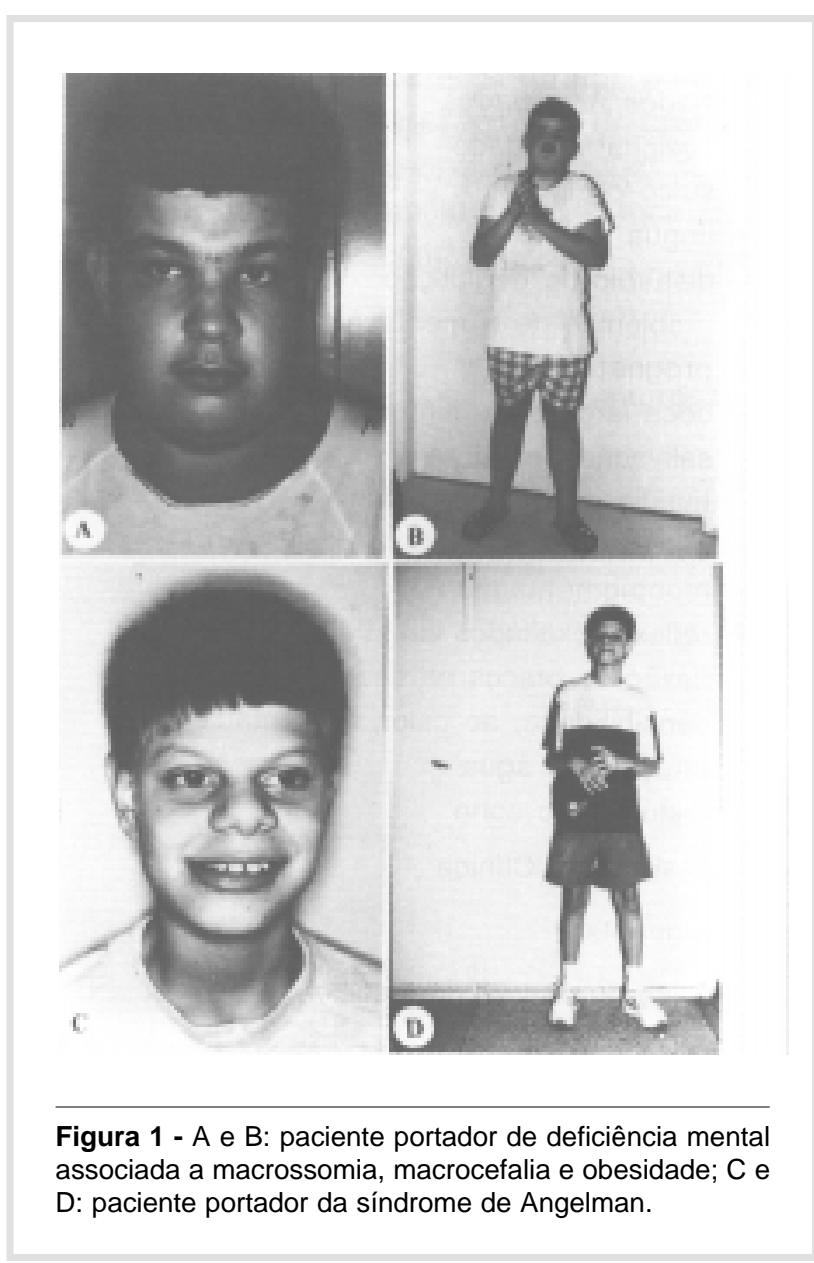


Tabela I - Estudo clínico, citogenético e molecular do paciente com suspeita da SA

Características clínicas, citogenéticas e moleculares

Idade

12 anos e 9 meses

Sexo

Masculino

Desenvolvimento e Achados Laboratoriais

- história pré-natal e neonatal normais

- ADNPM*

- ausência de involução neuromotora

- investigação metabólica normal

- cérebro estruturalmente normal

Achados Consistentes

- deficiência mental severa

- deficiência na fala

- movimentos atáxicos

- comportamento típico*

Achados Freqüentes

- Microcefalia

- Convulsões

- EEG anormal

Achados Associados

- occipital achatado

- sulco occipital

- língua protrusa

- distúrbio de deglutição

- problemas de alimentação, na infância

- prognatismo

- boca larga com dentes espaçados

- salivação freqüente

- mastigação exagerada

- estrabismo

- hipopigmentação

- reflexos exaltados em MMII

- flexão de braços na deambulação

- sensibilidade, ao calor, aumentada

- atração por água

- distúrbio do sono

Classificação Clínica

Citogenética

Análise do padrão de metilação

Análise da segregação de repetições de (CA)n

Biparental

Conclusão final

AS com herança biparental

assim como a determinação de riscos de recorrência. E, mais importante ainda, a definição do quadro clínico oferece subsídios para a intervenção de equipes multidisciplinares com a criança afetada e seus familiares, buscando a atenuação do quadro de base do distúrbio autista.

Neste trabalho, observamos que os dezessete indivíduos estudados apresentaram cariótipo com bandamento GTG normais, e a avaliação citogenética para a fragilidade do cromossomo $\mathrm{X}$ também foi normal. Entretanto, foram encontrados três casos (19\%) com fatores orgânicos relacionados aos problemas de comportamento e de aprendizado apresentados pelos pacientes.

Observamos um caso com diagnóstico clínico compatível com macrocefalia. A macrocefalia é definida como qualquer aumento de tamanho e de peso do cérebro, independente do comprometimento das funções neurológicas. Assim, um indivíduo é portador de macrocefalia, se a circunferência occipitofrontal exceder a média para idade e sexo em mais que dois desvios-padrão ${ }^{(24)}$. Estima-se que a incidência da macrocefalia seja de $2 \%$ a $6 \%$ na população geral $^{(25)}$, sendo observada a preponderância da macrocefalia em homens: $88 \mathrm{H} / 21 \mathrm{M}^{(25)}, 14 \mathrm{H} / 4 \mathrm{M}^{(26)}$ e $3 \mathrm{H} / 2 \mathrm{M}^{(27)}$. A macrocefalia compreende dois tipos principais: macrocefalia familial benigna e macrocefalia ou megaloencefalia.

Platt \& Nash ${ }^{(28)}$, Asch \& Myers ${ }^{(29)}$ e Day $\&$ Schutt ${ }^{(30)}$ descreveram os primeiros casos de macrocefalia familial benigna. Eles estudaram famílias que apresentavam macrocefalia em mais de uma geração e todos os portadores não apresentavam deficiência mental ou distúrbios neuropsicomotores. Foram evidenciadas algumas características clínicas, associadas à macrocefalia, nesses indivíduos: estreitamento biparietal, dolicocefalia, face quadrada, bossa frontal, hipoplasia de face média, filtro longo e os estudos de neuroimagem revelaram aumento dos ventrículos. Asch \& Myers ${ }^{(29)}$ sugeriram padrão de herança autossômica dominante, limitada aos homens, já que eles descreveram a macrocefalia em cinco homens, em duas gerações e um tio dos indivíduos da primeira geração também apresentava macrocefalia. 
Quanto à macrocefalia ou megaloencefalia, Walsh, em $1957^{(31)}$, descreveu duas irmãs e um irmão portadores de macrocefalia, embora os pais fossem normais e não consangüíneos, e descreveu outra família, na qual, os dois irmãos eram macrocefálicos e os pais normais. Nessas famílias, estavam associadas à macrocefalia, deficiência mental, atrofia da área precentral do cérebro, atrofia óptica, face grosseira, hipotrofia da musculatura esquelética e hipoplasia de medula adrenal. Além de deficiência mental, o portador de macrocefalia apresenta, freqüentemente, distúrbios neurológicos, como atraso do desenvolvimento neuropsicomotor, atraso do desenvolvimento da fala, hipotonia e convulsões ${ }^{(25)}$.

Quanto ao padrão de herança, De Almeida $\&$ De Barros $^{(32)}$ observaram casos com consangüinidade parental; Waisman ${ }^{(33)}$ sugeriu a possibilidade de herança ligada ao X e Fryns et al. ${ }^{34}$ sugeriram padrão autossômico dominante.

O paciente aqui estudado foi diagnosticado como portador de macrocefalia. Ele apresenta perímetro cefálico aumentado e o exame de raios-X evidenciou macrocrania com módulo aumentado. Além disso, apresentou deficiência mental e distúrbios de comportamento e aprendizado, sendo essas características encontradas em casos de macrocefalia. Quanto ao padrão de herança, como os pais são normais e não consangüíneos, o modelo de herança mais compatível seria o da macrocefalia não benigna, ligada ao X, recessiva.

Observamos que um dos casos estudados apresentou deficiência mental, macrossomia, macrocefalia, obesidade e algumas dismorfias. Em 1987, Baraitser et al. ${ }^{(23)}$ descreveram uma família, na qual, a mãe e dois filhos apresentavam deficiência mental, macrocefalia, obesidade e aparência facial similar. Em 1995, Baraitser et al. ${ }^{(35)}$ estudaram uma outra família, com dois primos afetados, apresentando deficiência mental, macrocefalia, obesidade e as mesmas dismorfias faciais apresentadas anteriormente. Essa nova forma de deficiência mental foi, então, definida como deficiência mental ligada ao cromossomo $\mathrm{X}$, associada à macrossomia, macrocefalia e obesidade.

O paciente estudado foi diagnosticado como afetado por essa nova síndrome de deficiência mental e anomalias congênitas múltiplas, já que apresentou as três características principais (deficiência mental, macrocefalia com macrossomia e obesidade) e a face típica, com as dismorfias que são descritas pela literatura (Tabela II).

Observamos um caso da SA, cujo cariótipo também se apresentou normal, porque esta síndrome é causada por alterações na região 15q11-13 do cromossomo 15, derivado da mãe, e essas alterações somente são detectadas, citogeneticamente, pela técnica de FISH (hibridação in situ fluorescente).

\begin{tabular}{|c|c|c|c|}
\hline Características Clínicas & Baraitser et al. (1987) & Baraitser et al. (1995) & Nosso Paciente \\
\hline Deficiência Mental & $2 / 2$ & $2 / 2$ & + \\
\hline Macrocefalia & $2 / 2$ & $2 / 2$ & + \\
\hline Frontal Quadrado & $2 / 2$ & $2 / 2$ & + \\
\hline Cristas supra-orbitárias proeminentes & $2 / 2$ & $2 / 2$ & + \\
\hline Hipertelorismo & $0 / 2$ & $0 / 2$ & - \\
\hline Ponta nasal larga & $2 / 2$ & $0 / 2$ & - \\
\hline Lábio superior proeminente & $2 / 2$ & $2 / 2$ & + \\
\hline Orelhas grandes & $2 / 2$ & $2 / 2$ & + \\
\hline Separação dos incisivos centrais & $1 / 2$ & $2 / 2$ & + \\
\hline Obesidade & $2 / 2$ & $2 / 2$ & + \\
\hline Macroorquidismo & $2 / 2$ & 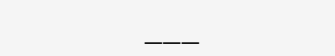 & + \\
\hline Alta estatura & $2 / 2$ & $2 / 2$ & + \\
\hline
\end{tabular}


A SA foi descrita por Angelman, em $1965^{(36)}$, e possui incidência de $1 \mathrm{em} 20000$ nascimentos $^{(37)}$. A SA é caracterizada, clinicamente, por hipotonia congênita central, microcefalia com achatamento acentuado de occipital, prognatismo, língua protrusa, ataxia de marcha, risos imotivados, convulsões com alterações próprias no eletroencefalograma (EEG), atraso neuromotor e deficiência mental severa, com ausência completa de fala.

Para confirmarmos o diagnóstico da SA, realizamos testes moleculares que detectam as alterações que ocorrem na região 15q11-13, materna, e levam à ocorrência da SA. Tais alterações podem ser: $73 \%$ dos casos são deleções na região 15q11-13 do cromossomo 15 , herdado da mãe; $3 \%$ dos casos são dissomia uniparental paterna; $5 \%$ dos casos são mutações no centro de imprinting da região 15q11-13, e $20 \%$ dos casos são de SA com herança biparental, sendo que, nesses casos, é que se concentram os casos familiais desta síndrome, com risco de recorrência de $50 \%{ }^{(38)}$.

Como os exames moleculares revelaram a presença dos alelos materno e paterno, excluímos a possibilidade de estar ocorrendo deleção materna ou dissomia uniparental paterna, e concluímos que este é um caso da SA com herança biparental. Nos casos da SA com herança biparental, a avaliação clínica se torna imprescindível para o diagnóstico correto. Ao avaliarmos, clinicamente, esse paciente, vimos que ele apresenta 26/28 dos critérios diagnósticos para a SA (Tabela I), incluindo o EEG típico da SA, sendo, portanto, considerado como um caso de SA com herança biparental. Em relação ao aconselhamento genético, concluímos que existiria risco de recorrência da ordem de $50 \%$ para a mãe, no caso de novas gestações.

Tendo em vista a freqüente associação da Síndrome do X-frágil com quadros de autismo infantil, realizamos o estudo citogenético para a pesquisa do $\mathrm{X}$-frágil. Não encontramos nenhum caso da Síndrome do X-frágil, talvez pelo número amostral pequeno $(\mathrm{N}=17)$ ou, talvez, porque, dependendo dos critérios diagnósticos adotados, a freqüência da Síndrome do $\mathrm{X}$-frágil, em amostras de indivíduos com autismo, e a frequiência de autismo, em indivíduos com a Síndrome do $\mathrm{X}$-frágil, têm variado bastante. Assim, a magnitude desta associação não é exatamente conhecida e, em alguns casos, talvez possa ser explicada pelo acaso.

\section{AGRADECIMENTOS}

Agradecemos as famílias que participaram deste estudo, sem as quais não seria possível a realização deste trabalho, assim como a Dra. Margarida Midea Cuccovia e os membros da Associação dos Amigos dos Autistas de Ribeirão Preto (AMA-RP) pelo encaminhamento dos familiares. Agradecemos ao Departamento de Neuropsiquiatria, principalmente, às suas docentes, Dra. Carolina Funayama e Dra. Regina Fernandes, e ao Setor de Radiologia do HCFMRP-USP. Suporte financeiro: CNPq (Processo $\mathrm{n}^{\circ}$ 401694/91.7) e FAPESP (Processo nº 96/4510-6).

MOLFETTA GA; FÉLIX TM \& PINA NETO JM. Clinical, genetic and cytogenetic study of autistic children. Medicina, Ribeirão Preto, 30: 514-521, oct./dec. 1997.

ABSTRACT: Infantile autism is characterized by a typical behavior that may be caused by an organic disease or by an emotional disorder. The objective of the present genetic-clinical and cytogenetic study was to detect the presence of organic diseases, especially those of genetic etiology, that migth be related to the signs and symptons of autism presented by 17 boys who attended at the Ribeirão Preto Association for Autistic Children. We concluded that 14 individuals had no organic alterations that might be related to their clinical picture; one subject presented a clinical picture compatible with macrocephaly; one subject presented a clinical picture compatible with a new X-linked mental deficiency syndorme associated with macrossomy, macrocephaly and obesity, and one subject presented the Angelman Syndrome (AS). The study of Xq27.3 fragility was also normal in all cases, excluding the presence of Fragile $X$ syndrome in all subjects. The specific molecular study for the detection of AS revealed the presence of biparental inheritance for the markers used. Since the clinical aspects of this patient was extremely suggestive, we conclude that he represented a case of AS with biparental inheritance.

UNITERMS: Autism, Infantile. Mental Retardation. Obesity. Fragile X Syndrome. Angelman Syndrome. 


\section{REFERÊNCIAS BIBLIOGRÁFICAS}

1 - KANNER L. Autistic disturbances of affective contact. Nerv Child 2: 217-250, 1943.

2 - KANNER L. Early infantile autism. J Pediatr 25: 211-217, 1944.

3 - ROSENBERG R. Autismo: histórico e conceito atual. Temas Desenv. Ano 1 (1): 4-7, 1991.

4 - SCHWARTZMAN JS. Autismo infantil. Temas Desenv. Ano 2 (10): 3-28, 1993.

5 - GRÜSPIM H. Autismo e psicose na infância. In: CAETANO D; FROTA-PESSOAO \& BECHELLI LPC. Esquizofrenia: atualização em diagnóstico e tratamento. Ed. Atheneu, Rio de Janeiro, cap. 6, p. 63-81, 1993.

6 - BRUNONI D. Autismo infantil: aspectos genéticos. Temas Desenv Ano 1 (5): 3-7, 1992.

7 - FOLSTEIN S \& RUTTER M. Autism: familial aggregation and genetic implications. J Autism Dev Disord 18: 3-30, 1988.

8 - FOLSTEIN S \& RUTTER M. Infantile autism: a genetic study of 21 twins pairs. J Child Psychiatry 18: 297-321, 1977.

9 - RITVO ER et al. Concordance for the syndrome of autism in 40 pairs of affected twins. Am J Psychiatry 142: 74-77, 1985.

10 - BAILEY A et al. Autism as strongly genetic disorder: evidence from a British twin study. Psychiatr Med 25: 63-77, 1995.

11 - ASSUMPÇÃO JUNIOR FB. Conceito e classificação das síndromes autisticas. In: SCHWATZMAN JS \& ASSUMPÇÃO JUNIOR FB. Autismo infantil. Memnon Edições Científicas, São Paulo, cap. 1, p. 3-16, 1995.

12 - TURK J. The fragile $X$ syndrome: on the way to a behavioral phenothype. Br J Psychiatry 160: 24-35, 1992.

13 - WATSON MS et al. Fragile $X$ in a survey of 75 autistic males. N Engl J Med 310: 1462, 1984.

14 - BLOMQUIST HK et al. Frequency of the fragile $X$ syndrome in infantile autism. Clin Genet 27: 113-117, 1985.

15 - BROWN WT et al. Fragile $X$ syndrome and autism: a multicenter study. Am J Med Genet 23: 341-352, 1986.

16 - TRANEBJAERG L \& KURE P. Prevalence of fra $(X)$ and other specific diagnosis in autistic individuals in a Danish Country. Am J Med Genet 38: 212-214, 1991.

17 - COHEN IL et al. Fragile $X$ syndrome in females with autism (letter). Am J Med Genet 34: 302-303, 1989.

18 - FÉLIX TM. Estudo clínico-citogenético na Síndrome do XFrágil. Tese de Mestrado, Faculdade de Medicina de Ribeirão Preto da USP, Ribeirão Preto, p. 1-138, 1995.

19 - MOORHEAD PS et al. Chromossome preparation of leukocytis cultures from peripheral bood. Exp Cell Res 20: 613-616, 1960.

20 - SCHERES JM. Human chromosome banding. Lancet I: 849, 1972.
21 - JACKY PB et al. Guidelines for the preparation and analysis of the fragile $X$ chromosome in lymphocytes. Am $\mathbf{J}$ Med Genet 38: 400-403, 1991.

22 - PINA NETO JM et al. Estudo clínico, citogenético e molecular nas síndromes de Prader-Willi e Angelman. Medicina, Ribeirão Preto 29: 488-495, 1996.

23 - BARAITSER M \& CLARK RD. A new X-linked mental retardation syndrome. Am J Med Genet 26: 13-15, 1987.

24 - DEMYER W. Megalencephaly in children. Neurology 22: 634-643, 1972.

25 - LORBER J \& PRIESTLY BL. Children with large heads: a practical appproach to diagnosis in 557 children, with special reference to 109 children with megalencephaly. Dev Med Child Neurol 23: 494-504, 1981.

26 - DE MYER W. Megalencephaly. In: VINKEN PJ, BRUYN GW. Handbook of clinical neurology. North Holland Biochemical Press, Amsterdan; v. 30, p. 684-693, 1981.

27 - DEKABAN AS \& SAKURAGAWA N. Megalencephaly. In VINKEN PJ \& BRUYN GW. Handbook of clinical neurology. North Holland Biochemical Press, Amsterdan, v. 30, p. 647-660, 1981.

28 - PLATT M \& NASHA. Benign familial megalencephaly. Pediatr Res 6: 426, 1972.

29 - ASCH AJ \& MYERS GJ. Benign familial macrocephaly: report of a family and review of the literature. Pediatrics 57: 535-539, 1976

30 - DAY RE \& SHUTT WH. Normal children with large heads-benign familial megalencephaly. Arch Dis Child 54: 512-517, 1979.

31 - WALSH FB. Megalencephaly. In: Clinical neuro-ophthalmology. $2^{\text {th }}$ ed. Williams \& Wilkins, Baltimore, p. 402-404, 1957.

32 - DE ALMEIDA G \& DE BARROS N. Megalencefalia: consideraciones a respecto de 7 casos diagnosticados en vida. Arq Neuropsiquiatr 22: 25, 1964.

33 - WAISMAN HA. Madison, Wis., 1967. Personal Communication.

34 - FRYNS JP et al. Mental retardation, macrocephaly, short stature and craniofacial dysmorphism in three sisters: a new entity among the mental retardation-macrocephaly syndromes? Clin Genet 33: 293-298, 1988.

35 - BARAITSER M; REARDON W \& VIJERATNAM S. Nonspecific $X$-linked mental retardation with macrocephaly and obesity: a further family. Am J Med Genet 57: 380-384, 1995.

36 - ANGELMAN H. Puppet children. Dev Med Child Neurol 7 : $681-688,1965$

37 - CLAYTON-SMITH J \& PEMBREY ME. Angelman Syndrome. J Med Genet 29: 412-415,1992.

38 - CHAN C-TJ et al. Molecular mechanisms in Angelman Syndrome: a survey of 93 patients. J Med Genet 30: 895-902, 1993.

Recebido para publicação em 30/06/97

Aprovado para publicação em 07/10/97 